SLAC-PUB-10299

astro-ph/0312183

January 2004

\title{
Testing the Cosmological Constant as a Candidate for Dark Energy
}

\author{
Jan Kratochvil, Andrei Linde \\ Department of Physics, Stanford University, Stanford, CA 94305-4060, USA \\ Eric V. Linder \\ Physics Division, Lawrence Berkeley National Laboratory, Berkeley, California 94720, USA \\ Marina Shmakova \\ Stanford Linear Accelerator Center, Stanford University, Stanford CA 94309, USA
}

\begin{abstract}
It may be difficult to single out the best model of dark energy on the basis of the existing and planned cosmological observations, because the parameter space of many different models can lead to similar observational consequences. However, each particular model can be studied and either found consistent with observations or ruled out. In this paper, we concentrate on the possibility to test and rule out the simplest and by far the most popular of the models of dark energy, the theory described by general relativity with positive vacuum energy (the cosmological constant). We evaluate the conditions under which this model could be ruled out by the future observations made by the Supernova/Acceleration Probe SNAP (both for supernovae and weak lensing) and by the Planck Surveyor cosmic microwave background satellite.
\end{abstract}

PACS numbers: $98.80 . \mathrm{Cq}, 11.25 .-\mathrm{w}, 04.65 .+\mathrm{e}$

\section{INTRODUCTION}

The observed present acceleration of the expansion of the universe [1, 2, 3] is commonly attributed to the presence of dark energy throughout the universe and gives rise to the question of what this dark energy actually is. There exist many different models of dark energy. Some of them are based on a particular choice of the scalar field potential [4, 5, 6], whereas some other models are based on certain modifications of general relativity, see e.g. 7] and references therein. Many of these models can have very similar observational consequences for certain choices of their parameters. Therefore it would be extremely hard to determine exactly which model, if any, is correct. This has led some authors to express a rather pessimistic attitude towards the observational investigation of dark energy [8].

However, one can look at this issue from a different perspective. Instead of trying to find which model of dark energy is correct, one can try to find which models can be ruled out by the existing and planned observations. This goal is quite realistic, and it can bring us extremely important information about the fundamental physics.

One particular case is especially interesting: the theory of dark energy based on general relativity with a constant vacuum energy $\Lambda>0$ (positive cosmological constant). First of all, the $\Lambda$ CDM model is by far the simplest dark energy model. In this model the dark energy remains constant, with the equation of state, or pressure to density ratio, $w \equiv p / \rho=-1$. Moreover, at present this is the only known dark energy model related to $\mathrm{M} /$ string theory [9]. Whereas it is quite possible that $\mathrm{M} / \mathrm{string}$ theory can describe quintessence with a time-dependent equation of state, all existing models of this type have problems describing stable compactifications of internal dimensions; see a discussion of this issue in [9, 10, 11].

An independent argument in favor of the simple cosmological constant model is obtained if one tries to find an explanation of the anomalous smallness of the dark energy density $\Lambda$ (the cosmological constant problem). A possible solution to this problem can be found in the context of the theory of eternal inflation if one replaces the cosmological constant by the theory of dark energy with a flat potential and uses the anthropic principle [5, 12, 13, 14]. However, in the simplest versions of this scenario the slope of the potential is expected to be so small that all observational consequences of this theory should be indistinguishable from the theory with a constant vacuum energy density [15].

An additional reason to be interested in the possible time evolution of dark energy is related to inflationary cosmology. Acceleration of the universe with timeindependent vacuum energy density is similar to the old inflation in the false vacuum state, whereas acceleration in the universe with the time dependent vacuum energy is similar to the standard slow-roll inflation. If we find that the universe experiences a stage of slow-roll inflation right now, this would make the slow-roll inflation in the early universe even more plausible.

On the other hand, if we do not find any observable deviations from the predictions of the simplest $\Lambda \mathrm{CDM}$ model, this will make all models of the dynamically changing dark energy much less attractive.

Therefore in this paper, instead of studying dark energy models in general, we will concentrate on a single well defined issue: What kind of observations could rule out the simple $\Lambda \mathrm{CDM}$ model? An answer to this ques- 
tion may help us in planning future experiments which would be specifically optimized for testing the simplest cosmological constant model.

The leading edge for dark energy exploration, ever since the current accelerating expansion of the universe was discovered [1], have been Type Ia supernovae observations, and they will acquire a new state-of-the-art instrument in the form of the Supernova/Acceleration Probe (SNAP) satellite 16]. SNAP will not only perform a distance-redshift measurement of some $2000 \mathrm{su}-$ pernovae (SNAP [SN]), but also conduct a wide field weak gravitational lensing survey (SNAP [WL]) 17], providing a complementary data set, as will at some point the ground-based LSST survey [18]. Additional measurements of the CMB, provided by the Planck Surveyor cosmic microwave background satellite [19], will help tighten the constraints obtained by SNAP.

In this paper, we will investigate a possibility to tighten the constraints on the parameters of dark energy and to test/rule out the simplest $\Lambda \mathrm{CDM}$ model using the results of these experiments. Our approach will be based on the methods developed in our previous paper [20].

\section{EXCLUDING THE COSMOLOGICAL CONSTANT}

The process of excluding the cosmological constant (or any other model) as a candidate for dark energy is achieved by mapping out the expansion history of the universe, i.e. the time-evolution of the scale factor $a(t)$. This is accomplished, for instance, by measuring the distance-redshift relation of Type Ia supernovae, which serve as "standard candles" in cosmology, having a known intrinsic luminosity normalizable through one parameter based on the width of their lightcurves, or flux vs. time relation. The proposed dedicated dark energy satellite mission SNAP, the Supernova/Acceleration Probe, will measure the precise luminosity distances to approximately 2000 such Type Ia supernovae within 2 years of its launch. The redshift range of these observed supernovae will span from $z=0.1$ out to $z=1.7$. In our calculations below, we bin the future data into 17 equally spaced redshift bins, and also include the expected $\sim 300$ supernovae from the presently running Nearby Supernovae Factory [21] in the bin with lowest redshift.

Complemented by the Planck mission, a satellite to observe the $\mathrm{CMB}$ at $z=1089$ and thus measure various cosmological parameters and breaking degeneracies in the SNAP observations, respectable constraints can be obtained on the equation of state of dark energy and its evolution with time.

SNAP's wide-field camera is not limited to studying supernovae, however. It will also be able to make use of the new, rapidly emerging observational method of weak gravitational lensing (SNAP [WL]) in mapping out the time evolution of the scale factor of the universe $a(t)$, including through the growth factor of large scale structure. Lensing will provide an independent measurement of the evolution history and through complementarity allow even tighter constraints.

Determining the equation of state is the crucial observational clue for the nature of dark energy. Commonly one defines the equation of state function $w(z)$ by

$$
p=w(z) \rho .
$$

In general, it is a function that can vary with time or redshift $z$. For pure vacuum energy, $w=-1$ independent of redshift.

To link observational data sets to existing physical models, it is useful to use a fitting function for $w(z)$ that contains only a few fitting parameters. The fewer parameters, the better their value can be constrained by a given observational data set, but - depending on the data - the less the resulting function might fit the actual data.

A widely applicable fit, combining the virtues of having only two fit parameters $\left(w_{0}\right.$ and $\left.w_{a}\right)$, yet fitting many theoretically conceivable scalar field potentials, especially in the slow roll regime, has been in use in cosmology for some time now, ever since its introduction in [22]:

$$
w(z)=w_{0}+w_{a} \frac{z}{1+z} .
$$

In particular it fits well small recent deviations from vacuum energy with $w=-1$. Most models of dark energy, except for some with heavily oscillating behavior like some PNGB models (cf. e.g. 23]) approach the limit of being barely distinguishable from the cosmological constant in a manner compatible with Linder's fit (2). For example, the linear potential treated in 20] shows a deviation from $w=-1$ to slightly higher values of $w$ only for $z \lesssim 1$. Of course, eventually in the future, once people have obtained the actual measurement data set, they will be able to compare it to the cosmological constant model directly, not having to revert to a fit first.

We take the SNAP baseline mission, as described in 24], including statistical and systematic errors amounting to a distance uncertainty of $1 \%$ at the depth $z=1.7$ of the survey. We marginalize over the absolute magnitude parameter $\mathcal{M}$, which includes the Hubble constant $H_{0}$, and over the dark energy density $\Omega_{D}$, where we assume the preferred value to be centered at $\Omega_{D}=0.72$, as favored by current observations (e.g. 3]). Complementary data from Planck, or from SNAP[WL], make it unnecessary to impose a prior on $\Omega_{D}$. The degeneracies are broken well enough to determine $\Omega_{D}$ with a precision comparable to a prior of $\sigma_{\Omega_{D}}=0.01$ [25].

The constraints from the data on the dark energy fit parameters $w_{0}$ and $w_{a}$ are analyzed within the Fisher matrix method 26, 27], providing probability ellipses at selected levels of confidence. Throughout this paper, we have chosen the $95 \%$ (or $2 \sigma$ ) confidence level. A much more detailed account on specifics of SNAP, its observational properties, errors and statistics, as well as a practical guide for reproduction of the Fisher matrix method used here, is given in [20] and references therein. 
Fig. 1 depicts the 95\% confidence level contour as obtained from SNAP [SN] and Planck, beyond which the cosmological constant can be excluded from being responsible for the dark energy density. If the SNAP supernovae measurements and the CMB results from the Planck mission select a point in this parameter space as being the most likely that lies outside of this contour, then the dark energy density causing the present acceleration of the expansion of the universe originates from something different than vacuum energy.

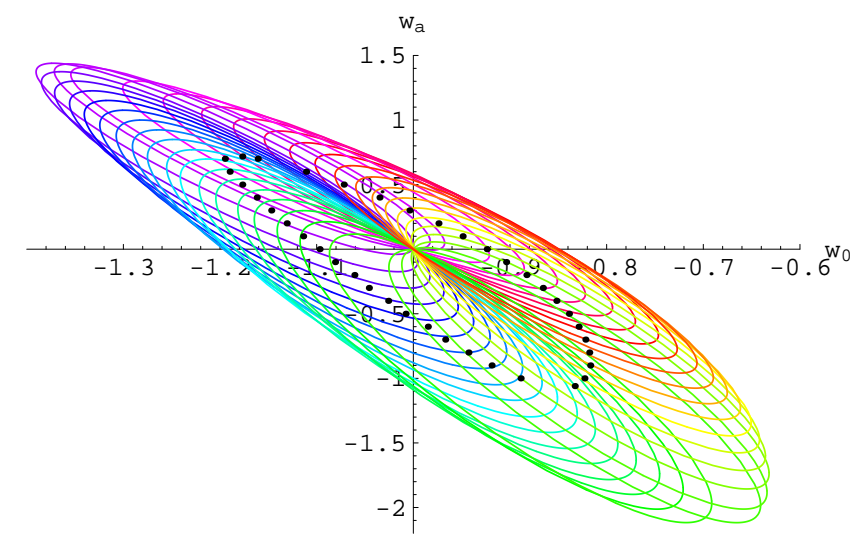

FIG. 1: 38 Fisher ellipses conspiring at 95\% confidence to outline the region beyond which the cosmological constant is ruled out as the main component of dark energy: if the most likely parameter value point from the combined SNAP [SN] and Planck measurement will lie outside the contour delineated by the centers (black dots) of the colored ellipses, we will have to abandon the idea of dark energy being due to pure vacuum energy with equation of state $p=-\rho$. This same black contour is also depicted by the outer (blue) curve in Fig. 2

Notice that all the ellipses in Fig. 1 exactly touch the cosmological constant point $\left(w_{0}=-1, w_{a}=0\right)$, the criterion for obtaining the contour. It is clear from the construction that the figure requires pointwise interpretation: as the Fisher ellipse is supposed to be centered around the actual (future) measurement point, not all the ellipses in the figure can be valid at the same time. Only one will be eventually, after the measurements will have been made, and in fact likely not even one that is drawn. What the graph tells us is that any point outside the contour, if the one most favored by the measurements, will have an associated ellipse not encompassing the cosmological constant point $\left(w_{0}=-1, w_{a}=0\right)$, thus enabling us to exclude the cosmological constant, at the $95 \%$ confidence level, as a possible cause of dark energy. Conversely, if the future measurement point will happen to lie inside the contour, we will be unable to rule out the cosmological constant, because the ellipse will encompass the point $(-1,0)$.

To clarify our methodology, let us note that instead of drawing many ellipses touching the point $w_{0}=-1$, $w_{a}=0$, and then connecting their central points, one could draw, as usual, one ellipse corresponding to the $2 \sigma$ deviation from this point. This would give an ellipse somewhat similar to the contour discussed above. However, this ellipse would be slightly different, and it would have a different interpretation. It would show us the points in the parameter space $\left(w_{0}, w_{a}\right)$ excluded at the $2 \sigma$ level by the observations favoring the simple cosmological constant model. This is not what we study in our paper.

Another comment should clarify the way we are using the parametrization $w_{0}, w_{a}$, related to the fit (2). This fit allows one to describe a broad class of the models of dark energy (including the simplest cosmological constant), but there might exist some exotic models for which this fit is inadequate. It is important to understand that when we will have the real data from SNAP and Planck, we will not need to use any fit anyway, as we may directly compare the data with the predictions of the simplest $\Lambda \mathrm{CDM}$ model. However, the use of the broadly applicable fit (2) allows one to obtain a very good idea of the results that can be obtained by various experiments.

In particular, SNAP will also carry out a wide field weak gravitational lensing survey. Measurements of the distortion of distant source shapes by intervening gravitational potentials probe the cosmology through both geometric effects on distances and the growth of large scale structure. Fig. 2] adds the expected constraints from this weak lensing information (see Appendix for more details) to the supernova and cosmic microwave background data.

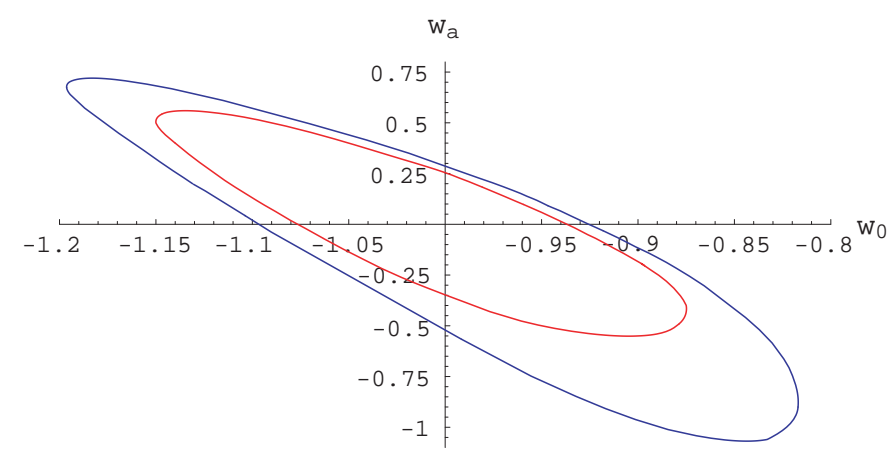

FIG. 2: The contours outside of which the cosmological constant can be excluded as the dominant contribution to dark energy. The outer contour (blue) was obtained by taking into account the observational systematic and statistical uncertainties of SNAP [SN] and Planck. For the inner contour (red), additionally SNAP [WL] was included.

Due to complementarity in the parameter dependencies, addition of weak lensing data can significantly improve constraints and narrow the region compatible with the cosmological constant. Fig. 2 shows the 95\% confidence level contour defined in Fig. 1 (outer, blue curve) by the ensemble of possible measurement realizations and also the corresponding (inner, red) curve when supplemented with weak lensing information. The phase space consistent with the cosmological constant is reduced by a 
factor of two. These data should be available within the next 10 years. Even tighter constraints may be possible by including other future cosmological observations, such as LSST's nearly full sky weak lensing survey a few years later [28].

A new, innovative method that has recently been explored in the context of constraining $w(z)$ [29], is crosscorrelating CMB anisotropies with the matter power spectrum. However, as pointed out in 29], the corresponding error bars today are still far too big. It is not obvious whether cosmic variance does not spoil the results obtained by this method irrecoverably [30]. This will require further investigation.

\section{CONCLUSIONS}

In a time when theoretical attempts at explaining the value of $\Lambda$ have so far been of limited success, apart from anthropic arguments [5, 12, 13, 14], it is more important than ever to take the observational clues we will have at our disposal to discriminate whether vacuum energy poses the dominant contribution to the dark energy, or whether different physics resides at its core.

It is an overzealous ambition to expect from present and future observational data that they will direct us unambiguously towards a unique dark energy model. Yet, we can test each of the models and rule out many of them. As we have shown in this paper, within a decade, we shall gain the possibility to test and maybe even rule out the most traditional of all dark energy models, the cosmological constant model.

This model is by far the simplest of all models of dark energy. Moreover, even though in the future we may learn how various dark energy models could be related to string theory and M-theory, at present the cosmological constant model remains the only one for which this possibility was actually demonstrated [9]. Therefore measurements ruling out the cosmological constant would have profound implications for particle physics.

Meanwhile, measurements consistent with a cosmological constant model will not resolve the mystery of the underlying physics - whether it is a pure vacuum energy or a more complex extension of physics merely possessing parameter values close to the cosmological constant. But at least in some of these cases (as shown in 20]) we will have a grace period of tens of billions of years to resolve the issue.

As a program for the future, for the planning of observations exploring the nature of dark energy, we would like to stress that it is most important to realize the benefit obtained from the interplay of various different observation missions and techniques. Degeneracies inherent in individual observation methods are broken efficiently by considering several different ones. If one takes, for instance, SNAP supernovae alone (contours not depicted in this paper, but see [20] for ellipses for that data set alone), the constraints are informative, yet they gain a tremendous improvement from including the Planck data coming from the measurements of the CMB, which will be available around the same time as SNAP. Quite a further impressive improvement is achieved if weak lensing from SNAP — obtained by the same mission, but with a different technique - is added. And another, equally impressive improvement, although some years later than the above, is expected to come from including LSST, a ground-based, nearly full sky weak lensing survey.

As we see, a lot of work to be done lies ahead, and only the joint effort of a diversity of space- and groundbased observations, combined with an innovative use and analysis of the data gathered by these instruments, will provide us with the best possible information on the nature of dark energy, fundamental cosmological physics, and thereby with knowledge about the future evolution and ultimate fate of our universe.

It is a pleasure to thank Niayesh Afshordi, Gary Bernstein, JoAnne Hewett, and Anthony Tyson for useful discussions. The work by J.K. was supported by the Stanford Graduate Fellowship and the Sunburst Fund of the Swiss Federal Institutes of Technology (ETH Zurich and EPFL). The work by A.L. was supported by NSF grant PHY-0244728. The work by M.S. was supported by DOE grant DE-AC03-76SF00515. The work by E.L. was supported in part by the Director, Office of Science, DOE under DE-AC03-76SF00098 at LBL.

\section{APPENDIX}

\section{A. Weak Lensing with SNAP}

Gravitational lensing involves the deflection of light rays from distant sources by the intervening gravitational potentials of, e.g., large scale structures of matter. One can relate the power spectrum of matter density fluctuations to the distribution of image distortions (shear) and size magnifications (convergence), weighted by distance dependent strength or focusing factors. Weak lensing refers to the regime where these effects are small and observed statistically rather than through more obvious multiple imaging, for example. Thus measurements of weak lensing probe the dark energy through the expansion history, both its effect on distances and the suppression of growth of matter structure as dark energy dominates and the expansion accelerates.

Numerous methods involving weak lensing are actively being investigated to develop an optimal probe of the nature of dark energy. The situation is still uncertain: only recently have computations included a time variation such as $w_{a}$ and systematic effects are not well identified. Here we take a greatly simplified version of the most 
promising method for reducing the dominant systematic error - cross-correlation cosmography [31, 32].

This involves studying background sources at different redshifts relative to the same foreground mass screen, allowing cancellation of many issues such as spurious distortion due to the instrumental point spread function and ignorance of the exact lensing mass distribution. This method comes close to providing a pure geometric test of the ratio between comoving distances to the various source redshifts. While the true situation is more complex, for the calculation in this paper we take SNAP wide field data to provide determination of the ratio

$$
R \equiv \frac{r_{s 1}-r_{l}}{r_{s 2}-r_{l}}
$$

where $r_{l}$ is the comoving distance to the lens and $r_{s i}$ is the comoving distance to the $i$ th source plane, to $0.2 \%$ at three lens redshifts $z_{l}=0.3,0.6,0.9$. Furthermore we fix the sources to lie in planes at $z_{s 2}=2 z_{s 1}=4 z_{l}$. This is clearly a toy model but it succeeds in reproducing the more sophisticated parameter estimation contours in Fig. 2 of [31]. Thus this approach provides a readily calculable first step toward including forthcoming weak lensing data.
[1] R.A. Knop et al., "New Constraints on $\Omega_{M}, \Omega_{\Lambda}$, and $\mathrm{w}$ from an Independent Set of Eleven HighRedshift Supernovae Observed with HST", ApJ in press astro-ph/0309368; J.L. Tonry et al., "Cosmological Results from High-z Supernovae", Astrophys.J. 594, 1 (2003) astro-ph/0305008; S. Perlmutter et al., "Measurements of Omega and Lambda from 42 High-Redshift Supernovae," Astrophys. J. 517, 565 (1999) astro-ph/9812133, see also http://snap.lbl.gov A. G. Riess et al., "Observational Evidence from Supernovae for an Accelerating Universe and a Cosmological Constant," Astron. J. 116, 1009 (1998) astro-ph/9805201.

[2] J. L. Sievers et al., "Cosmological Parameters from Cosmic Background Imager Observations and Comparisons with BOOMERANG, DASI, and MAXIMA," astro-ph/0205387 J. R. Bond et al., "The cosmic microwave background and inflation, then and now," arXiv astro-ph/0210007

[3] D. N. Spergel et al., "First Year Wilkinson Microwave Anisotropy Probe (WMAP) Observations: Determination of Cosmological Parameters," arXiv astro-ph/0302209

[4] T. Banks, "T C P, Quantum Gravity, The Cosmological Constant And All That..," Nucl. Phys. B 249, 332 (1985).

[5] A.D. Linde, "Inflation And Quantum Cosmology," Print86-0888 (June 1986), in: Three hundred years of gravitation, (Eds.: Hawking, S.W. and Israel, W., Cambridge Univ. Press, 1987), 604-630.

[6] C. Wetterich, "Cosmology And The Fate Of Dilatation Symmetry," Nucl. Phys. B 302, 668 (1988); P. G. Ferreira and M. Joyce, "Cosmology with a Primordial Scaling Field," Phys. Rev. D 58, 023503 (1998) [arXiv astro-ph/9711102]; B. Ratra and P. J. Peebles, "Cosmological Consequences Of A Rolling Homogeneous Scalar Field," Phys. Rev. D 37, 3406 (1988); I. Zlatev, L. M. Wang and P. J. Steinhardt, "Quintessence, Cosmic Coincidence, and the Cosmological Constant," Phys. Rev. Lett. 82, 896 (1999) [arXiv astro-ph/9807002.

[7] G. Dvali and M. S. Turner, "Dark energy as a modification of the Friedmann equation," arXiv astro-ph/0301510 S. Mukohyama and L. Randall, "A dynamical approach to the cosmological constant,"
arXiv hep-th/0306108 A. Lue, R. Scoccimarro and G. Starkman, "Differentiating between Modified Gravity and Dark Energy," arXiv astro-ph/0307034

[8] I. Maor, R. Brustein, J. McMahon and P. J. Steinhardt, "Measuring the Equation-of-state of the Universe: Pitfalls and Prospects," Phys. Rev. D 65, 123003 (2002) [arXiv astro-ph/0112526]; I. Maor and R. Brustein, "Distinguishing among scalar field models of dark energy," Phys. Rev. D 67, 103508 (2003) [arXiv hep-ph/0209203.

[9] S. Kachru, R. Kallosh, A. Linde and S. P. Trivedi, "De Sitter vacua in string theory," Phys. Rev. D 68, 046005 (2003) [arXiv hep-th/0301240]; C. P. Burgess, R. Kallosh and F. Quevedo, "de Sitter string vacua from supersymmetric D-terms," JHEP 0310, 056 (2003) [arXiv hep-th/0309187.

[10] R. Kallosh, A. Linde, S. Prokushkin and M. Shmakova, "Supergravity, dark energy and the fate of the universe," Phys. Rev. D 66, 123503 (2002) [arXiv hep-th/0208156].

[11] M. Gutperle, R. Kallosh and A. Linde, "M / string theory, S-branes and accelerating universe," JCAP 0307, 001 (2003) [arXiv hep-th/0304225.

[12] S. Weinberg, "Anthropic Bound On The Cosmological Constant," Phys. Rev. Lett. 59, 2607 (1987); S. Weinberg, "The cosmological constant problems," arXiv astro-ph/0005265

[13] J. Garriga and A. Vilenkin, "On likely values of the cosmological constant," Phys. Rev. D 61, 083502 (2000) [arXiv astro-ph/9908115].

[14] A. Linde, "Inflation, quantum cosmology and the anthropic principle," arXiv hep-th/0211048

[15] J. Garriga and A. Vilenkin, "Testable anthropic predictions for dark energy," Phys. Rev. D 67, 043503 (2003) [arXiv astro-ph/0210358; J. Garriga, A. Linde and A. Vilenkin, "Dark energy equation of state and anthropic selection," arXiv hep-th/0310034

[16] http://snap.lbl.gov

[17] A. Refregier et al. "Weak Lensing from Space III: Cosmological Parameters", submitted to ApJ, astro-ph/0304419

[18] http://www.lsst.org

[19] http://astro.estec.esa.nl/planck

[20] R. Kallosh, J. Kratochvil, A. Linde, E. V. Linder and M. Shmakova, "Observational Bounds on Cosmic Doomsday," JCAP 0310, 015 (2003) [arXiv astro-ph/0307185. 
[21] http://snfactory.lbl.gov

[22] E.V. Linder, "Exploring the Expansion History of the Universe", Phys. Rev. Lett. 90, 091301 (2003), astro-ph/0208512 ; E.V. Linder, "Probing Dark Energy with SNAP", in Proceedings of IDM2002, astro-ph/0210217

[23] J. Weller and A. Albrecht, "Future Supernovae observations as a probe of dark energy," Phys. Rev. D 65, 103512 (2002) [arXiv astro-ph/0106079.

[24] A.G. Kim, E.V. Linder, R. Miquel, and N. Mostek, "Effects of Systematic Uncertainties on the Supernova Determination of Cosmological Parameters", MNRAS in press, astro-ph/0304509

[25] J.A. Frieman, D. Huterer, E.V. Linder, and M.S. Turner, "Probing Dark Energy with Supernovae: Exploiting Complementarity with the Cosmic Microwave Background", Phys. Rev. D67, 083505 (2003), astro-ph/0208100

[26] M. Tegmark, D.J. Eisenstein, W. Hu, and R. Kron, "Cos- mic Complementarity: Probing the Acceleration of the Universe", astro-ph/9805117

[27] M. Tegmark, A.N. Taylor, and A.F. Heavens, "Karhunen-Loève eigenvalue problems in cosmology", ApJ 480, 22 (1997).

[28] J. A. Tyson, private communication.

[29] J. Garriga, L. Pogosian and T. Vachaspati, "Forecasting Cosmic Doomsday from CMB/LSS Cross-Correlations," arXiv astro-ph/0311412

[30] N. Afshordi, private communication.

[31] G. M. Bernstein and B. Jain, "Dark Energy Constraints from Weak Lensing Cross-Correlation Cosmography", ApJ in press, arXiv astro-ph/0309332

[32] B. Jain and A. Taylor, "Cross-correlation Tomography: Measuring Dark Energy Evolution with Weak Lensing", Phys. Rev. Lett. 91 (2003) 141302 [arXiv astro-ph/0306046]. 